\title{
Is MRI Overutilized for Evaluation of Knee Pain in Veterans?
}

\author{
Scott Koenig, MD ${ }^{1}$ George Morcos, BA ${ }^{1}$ Rohan Gopinath, $\mathrm{MD}^{1}$ Kenneth Wang, MD, PhD ${ }^{2}$ \\ Frank Henn 3rd, MD ${ }^{1}$ Natalie L. Leong, MD $^{1}$ \\ 1 University of Maryland School of Medicine, Orthopaedics, Baltimore, \\ Maryland \\ 2 Department of Radiology, Veterans Affairs Commission, Baltimore, \\ Address for correspondence Natalie L. Leong, MD, 10 N. Greene \\ Street, Baltimore, MD 21201 (e-mail: natalie.leong@va.gov). \\ Maryland
}

J Knee Surg 2023;36:305-309.

\begin{abstract}
MRI is an essential diagnostic imaging modality for many knee conditions; however, it is not indicated in the setting of advanced knee arthritis. Inappropriate MRI imaging adds to health care costs and may delay definitive management for many patients. The primary purpose of this study was to ascertain the frequency of inappropriate MRI scans performed at one Veterans' Administration Medical Center (VAMC). We performed a retrospective chart review of all knee MRIs ordered over a 6-month period. Inappropriate MRI was defined as MRI performed prior to radiographs (XRs), or in the presence of XRs demonstrating severe osteoarthritis, without leading to a nonarthroplasty procedure of the knee. Of the 304 cases reviewed, 36.8\% (112) of the MRIs were deemed inappropriate, 33 were ordered by orthopedists, and 79 were ordered by other health care providers. Of the 33 ordered by orthopedists, 25 were ordered by retired/nonsurgical orthopedists. Obtaining an MRI delayed care by an average of 29.2 days. Of the 252 cases that had XR prior to MRI, none included all four views in the

Keywords

- MRI

- osteoarthritis

- knee pain

- veterans standard knee XR series and only four had weightbearing images. Over a third of knee MRIs performed at this VAMC were inappropriate and delayed care. Additionally, no XRs in our study contained all the necessary views to properly assess knee arthritis. These concerning findings signify a potential opportunity for education in diagnostic strategies, to better patient care and resource utilization in the VAMC.
\end{abstract}

Knee osteoarthritis is a highly prevalent disease in the US, and even more so among patients who are veterans, at least in part due to high physical demands on their knees while in the service. ${ }^{1,2}$ The prevalence and debilitating nature of osteoarthritis perpetuate its financial burden, and these costs will continue to rise as more total knee replacements are being performed and at an earlier age. ${ }^{3-5}$ Veterans, in particular, are more likely to report chronic joint symptoms and activity limitation than nonveterans, making improved efficiency in the use of health care resources devoted to this issue more critical. ${ }^{2}$

received

July 1,2020

accepted after revision

June 21, 2021

article published online

September 2, 2021
The Kellgren-Lawrence classification (KL classification) first described the radiographic changes characteristic of knee osteoarthritis over 50 years ago, and it is well-established that XRs are an effective means of diagnosing knee osteoarthritis. ${ }^{6,7}$ Although debates exist, the preferred radiographic views for evaluating knee osteoarthritis are a weight-bearing anteroposterior (AP) view, a posteroanterior (PA) flexion view, a lateral view, and a sunrise/merchant view. $^{8-10}$ MRI has improved diagnosis in the evaluation of certain patients with knee pain, but at the same time has notably increased health care spending. ${ }^{11,12}$ There has been

(c) 2021. Thieme. All rights reserved. Thieme Medical Publishers, Inc., 333 Seventh Avenue, 18th Floor. New York, NY 10001, USA
DOI https://doi.org/ 10.1055/s-0041-1733880. ISSN 1538-8506. 
Table 1 Patient demographics. Age and BMI are presented as a mean followed by 95\% confidence interval

\begin{tabular}{|l|l|l|l|l|}
\hline & Overall $(\boldsymbol{n}=\mathbf{3 0 6})$ & MRI before XR $(\boldsymbol{n}=\mathbf{5 4})$ & MRI after XR $(\boldsymbol{n}=\mathbf{1 8 3})$ & MRI and XR on same day $(\boldsymbol{n}=\mathbf{7})$ \\
\hline Sex $(\%$ male $)$ & 79.7 & 79.6 & 79.8 & 86 \\
\hline Age, years & $53.2(29.9-76.5)$ & $53.9(31.0-76.8)$ & $54.6(32.6-76.6)$ & $52.1(22.7-81.5)$ \\
\hline BMI & $31.6(20.0-43.2)$ & $30.6(25.5-35.7)$ & $31.4(20.0-42.8)$ & $36.1(28.3-43.9)$ \\
\hline
\end{tabular}

Abbreviations: BMI, body mass index; XR, radiographs.

increased study on the overutilization of MRI and "defensive medicine" in the past few years, and with good reason, as one study found that $40 \%$ of knee MRIs were deemed inappropriate for the diagnosis of the patient. ${ }^{13-16}$ Many findings on MRI of the knee, such as degenerative meniscal tears, are present in asymptomatic patients, bringing into question the utility of MRI in patients with known osteoarthritis. ${ }^{17,18}$ Furthermore, Song et al described MRI as "useful" in only $18 \%$ of the degenerative knee evaluations compared to $84 \%$ of the sports injury evaluations. ${ }^{19}$ Furthermore, knee MRI is not indicated in patients with XRs diagnostic of osteoarthritis, further supporting that XRs should be obtained prior to MRI in most cases. ${ }^{20}$ Time to acquisition of MRI should also be scrutinized, since MRI is not as readily accessible as XRs, and it has been shown to delay care of patients with anterior cruciate ligament tears by 89 days in one study in England. ${ }^{21}$

To our knowledge, this is the first study assessing the application of MRI in the evaluation of knee pain in veterans, focusing on inappropriate resource utilization and delay in care. The primary purpose of this retrospective study was to ascertain the number of inappropriately ordered knee MRIs performed in veterans, and to determine the delay in acquiring these MRIs. We suspected that MRI would be an overutilized diagnostic tool that delayed care in patients at VAMC. Additionally, we investigated the training of providers who ordered these MRIs, and the number of XRs that incorporated weight-bearing views and four views to adequately assess the three compartments of the knee. We hypothesize that MRI is an overutilized diagnostic tool in veterans with knee pain.

\section{Design}

Local institutional review board approval was obtained for this retrospective study (HP-00084062). The mPower radiology analytics platform (Nuance; Burlington, MA) was used to search for all knee MRI examinations performed at the Baltimore Veterans' Administration Medical Center (VAMC) from January 1, 2018, through June 30, 2018. We collected demographic information (shown in -Table 1) and determined if the MRI was obtained prior to or after XRs of 306 extremities. Fellowship-trained attending orthopedic surgeons graded the XRs using the KL grading scale, considering joint space narrowing, osteophytes, and subchondral sclerosis. We deemed MRIs inappropriate if they were 1) performed on an arthritic knee (KL 3 or 4) with no subsequent surgery or 2) prior to obtaining an XR with no subsequent surgery. In addition, we ascertained the specialty of the ordering provider, the number of days required for MRI completion from the time of the order, and whether the patient had any knee procedures prior to or after obtaining the MRI. The time to MRI completion was a surrogate in our study for delay in definitive care. The specialty of the ordering provider was categorized into two groups: orthopedists and nonorthopedic providers, which included primary care providers, other specialists, nurse practitioners, and physician assistants. We further divided orthopedic surgeons into operative and nonoperative orthopedic surgeons (the latter group consists of semiretired orthopedists who no longer perform surgery). Patients were excluded from the study if they had a prior total knee arthroplasty on the knee that underwent MRI scan. There were no prisoners, pregnant women, or children under the age of 18 in the cohort. Statistical analysis was performed with Chi-square testing, with statistical significance set at $p<0.05$, using JMP statistical analysis software.

\section{Results}

Analysis was performed on a total of 306 knees with MRIs (298 patients): 54 (17.6\%) had their MRI prior to knee XR, 183 (59.8\%) had their MRI after XR, and 7 (2.3\%) had the MRI and $\mathrm{XR}$ on the same day. The ordering providers were classified by specialty ( - Table 2 ). Primary care providers ordered the most knee MRIs in this study at 137 (44.8\%), followed by orthopedic surgeons at $112(36.6 \%)$, and then other health care providers at $55(18 \%)$.

Data from a total of 304 cases was reviewed; $37.1 \%$ (113) of the MRIs were deemed inappropriate. We were unable to ascertain the date of MRI for 2 of the knees, leaving 304 of the 306 MRIs available for data interpretation. MRI completion took 29.2 days on average with a standard deviation (SD) of 19.1 and median of 26 days ( $\mathbf{- F i g . ~} \mathbf{1}$ ). Of the 252 cases that obtained an XR prior to MRI, none included all four preferred views (AP, PA flexion view, lateral, and a sunrise/merchant view), while only four had weight-bearing images.

Table 2 Number of MRIs ordered by provider type

\begin{tabular}{|c|l|l|l|l|}
\hline Provider & $\begin{array}{l}\text { MRI } \\
\text { before } \\
\text { XR }\end{array}$ & $\begin{array}{l}\text { MRI } \\
\text { after } \\
\text { XR }\end{array}$ & $\begin{array}{l}\text { MRI and } \\
\text { XR same } \\
\text { day }\end{array}$ & Total \\
\hline Orthopaedists & 10 & 101 & 1 & 112 \\
\hline Surgical & 3 & 25 & 1 & 28 \\
\hline Nonsurgical/retired & 7 & 76 & 0 & 84 \\
\hline $\begin{array}{l}\text { Nonorthopaedic } \\
\text { providers }\end{array}$ & 43 & 143 & 6 & 192 \\
\hline
\end{tabular}




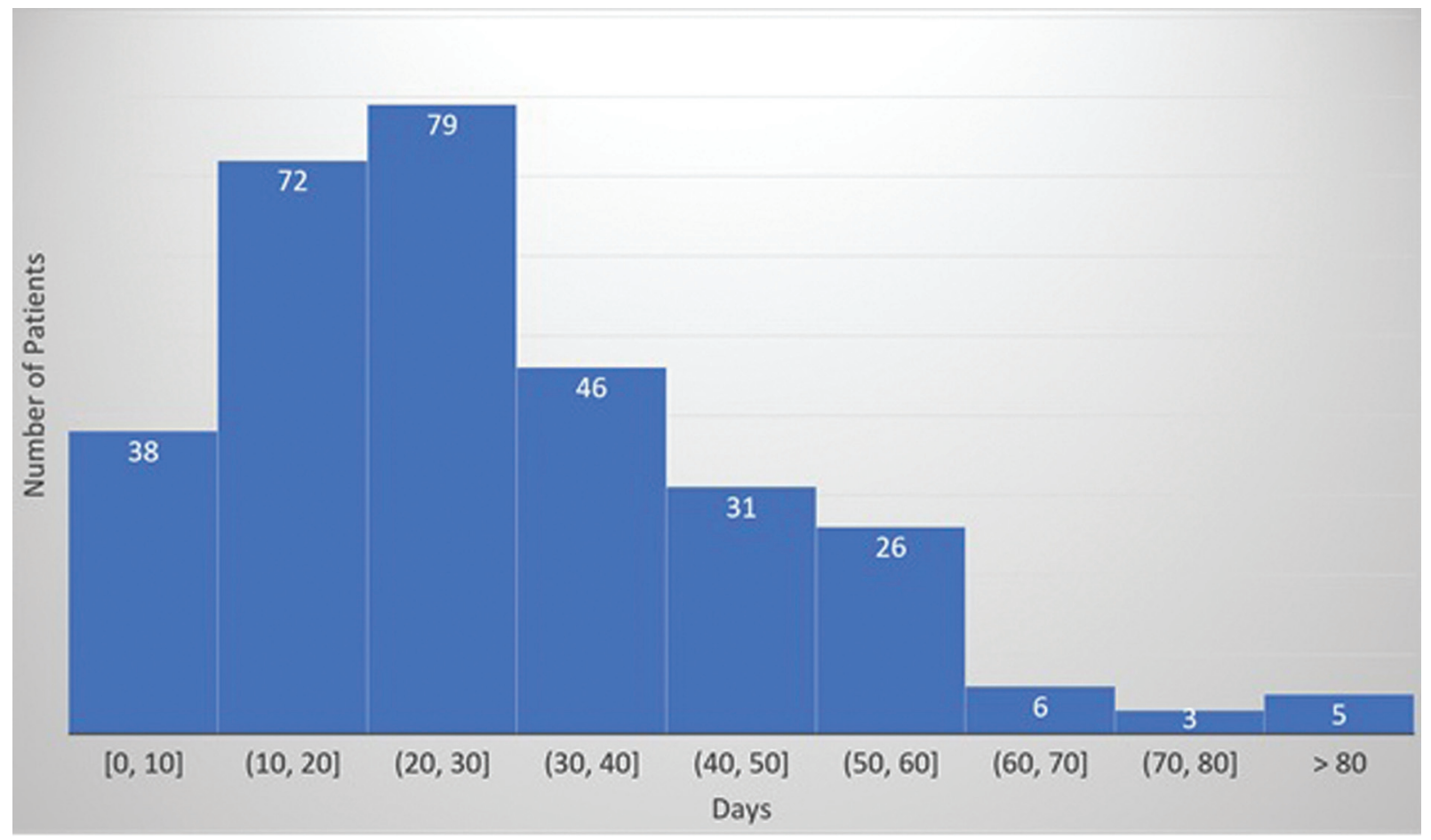

Fig. 1 Delay in care due to ordering of MRIs. Median $=26$ days; mean $=29.2$ days; standard deviation (SD) $=19.1$ days.

The distribution of providers ordering unnecessary MRIs can be found in - Fig. 2. There were 113 (37.1\%) MRIs deemed inappropriate; 46 patients had undergone an MRI without prior XR and no subsequent surgeries, and 67 patients had an arthritic knee (KL 3 or 4) without subsequent surgeries. Of those inappropriate MRIs, 33 (29.5\%) were ordered by orthopedic surgeons, while 79 (70.5\%) were ordered by nonorthopedic providers. There was one entry that lacked provider type in the system, so this data point was eliminated from this analysis, leaving 112 instead of 113 knees. A Chisquare test of goodness of fit was performed to examine the relationships between provider type and inappropriately ordering MRIs. The relationship between these variables was significant, $X 2(1, n=112)=18.893, p=0.00001$. Of

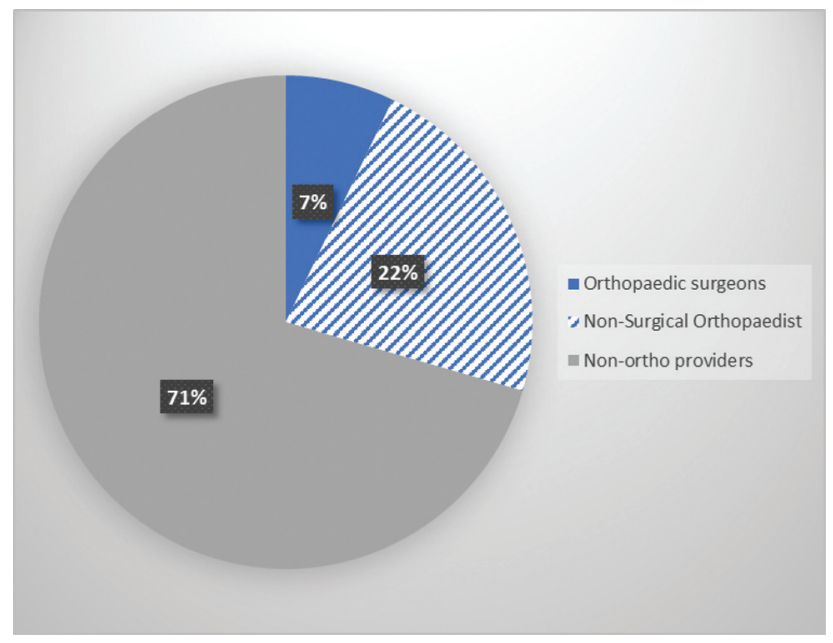

Fig. 2 Proportion of inappropriately ordered MRIs by provider type. the 33 ordered by orthopedic surgeons, 25 were ordered by orthopedic surgeons who are no longer surgically active. Another Chi-square test was performed, dividing orthopedists into surgical and nonsurgical; the relationship between provider type and inappropriately ordered MRIs was also found to be significant with a much lower $p$ value, X2 (1, $n=33$ ) $=8.758, p=0.003$. Of the total number of MRIs ordered, orthopedic surgeons ordered $29.4 \%$ (33) of the total number of unnecessary MRIs (112).

\section{Discussion}

To our knowledge, this study is the first to evaluate overuse of MRI in the evaluation of knee pain in the veteran population. Health care spending is becoming increasingly scrutinized, and the inappropriate use of MRI is contributing to higher orthopedic health care expenditures. ${ }^{22,23}$ Acute injury, effusion, and ligamentous instability in patients without osteoarthritis are considered appropriate indications for ordering knee MRIs, according to most orthopedic sports surgeons. ${ }^{24}$ Although the cost of MRI varies, one study noted a knee MRI costs around $\$ 459$ in Medicare and as high as $\$ 1,628$ in private settings. ${ }^{25}$ Reducing health care costs in VAMC specifically has been the subject of research in other fields of medicine. ${ }^{26-28}$ Our study hypothesis was strongly supported in this study, and reveals that the overuse of MRI may be a potential area VAMC can target to reduce health care expenditures and prevent delays in the care of orthopedic patients with knee pain.

Our results showed that $37.1 \%$ of veteran patients had knee MRIs either prior to any XRs or in the presence of known KL grade 3 or 4 osteoarthritis on radiographs. These 
are situations in which the MRI is unnecessary and are unlikely to change the treatment plan. These findings are significantly higher than the $13 \%$ inappropriate MRIs reported in the study by Sherman et al, suggesting the problem of MRI misuse in orthopedic knee pathology may be worse in the VAMC than the private setting. ${ }^{16}$ In part, this may be because MRI orders at the VAMC are not subject to insurance review such as by private insurers. In addition, a miniscule amount of the radiographs obtained were weightbearing and included all relevant views to properly assess knee osteoarthritis. This suggests there may be a lack of understanding among providers in how to properly evaluate knee osteoarthritis in this patient population. There is a high prevalence of osteoarthritis among veterans, and they tend to present for care with more chronic joint problems such as osteoarthritis, so it is all the more imperative that knee $\mathrm{OA}$ should be high on the differential and properly evaluated.

In our study, obtaining an MRI of the knee took an average of 29 days. Delays in access to health care amongst veterans has been the subject of national news in recent years. The national database research recently backed these comments, showing that $29 \%$ of American veterans delayed seeking care compared to $17.2 \%$ of civilian Americans, even after adjusting for regions and personal factors. ${ }^{29}$ Recognizing areas of improvement in the system can be the first step to real implemented changes that lead to progress as demonstrated at VAMC in Indiana. ${ }^{30}$ There have been others including the "Veteran's Choice program," a process by which the VAMC can offer non-VA health care services for eligible veterans to help facilitate care in situations of geographical constraints or overburdened VA facilities. ${ }^{31}$ For a population of patients which is already subject to delays in care, ordering inappropriate MRIs that perpetuate the delay is a problem that requires similar attention and actions. Our findings also support the results found by Bernstein et al, suggesting that nonorthopedic physicians may use knee MRI scans to screen normal or osteoarthritic knees for a diagnosis more often than orthopedic surgeons. ${ }^{32}$ Randomized controlled trials in the UK have investigated a similar topic, assessing if MRI referral by the general practitioner or emergency department is cost-effective in younger patients with traumatic knee pain. They similarly found that MRI was adding to health care costs due to physiotherapy sessions and arthroscopies without improving outcomes. ${ }^{33-35}$ The authors hoped to provide evidence in this environment of defensive medicine to support health care providers in following appropriate diagnostic practices, in order to stunt cost expenditures. Our study supports these beliefs in a different patient population but with the same goal.

There are several limitations to our study. The information obtained was from a single institution during a 6-month span. At our institution, the orthopedic intake clinic is primarily staffed by nonoperative providers, and may result in unique data patterns. It is worth noting that while nonoperative orthopedic providers ordered more unnecessary MRIs, the proportion of unnecessary MRIs to total MRIs $(30 \% ; 25 / 84)$ was very similar to that in surgical orthopedists (28\%; 8/28). This could be explained by the fact that nonop- erative orthopedic providers may see more "non-operative" or "pre-operative" knee pain complaints. The population of this study was from a single institution, which may lead to selection bias. Thus, a multicenter study would provide more information on the generalizability of our findings across VA hospitals. Additionally, there were veterans in our study who elected to participate in the Veteran's Choice program and transfer their care to private providers in the community. It was largely clear from notes provided by primary care physicians, physical therapists, and orthopedic surgeons if the patient eventually underwent surgery after the MRI, but we did not have direct access to non-VA provider notes from the program. The determination of osteoarthritis was based on the KL radiographic scale, which is subjective and therefore a limitation worth mentioning. ${ }^{36}$ Furthermore, further studies may be needed to evaluate the effect of social factors such as socioeconomic and insurance status. There is no funding to be disclosed for this study.

This study provides evidence that MRI is being overutilized for the assessment of knee pain and is likely a source of inefficient utilization of resources at our local VAMC. The data reveals that ordering MRI in the setting of advanced osteoarthritis is a topic that needs to be addressed through increased awareness and education. Future directions and guidelines should be developed to assist VAMC physicians with regard to appropriate criteria for ordering diagnostic imaging for the evaluation of knee pain, in an effort to reduce the number of inappropriately ordered MRIs.

\section{Conclusion}

A notable number of MRIs were inappropriately ordered at a VAMC and likely increased the costs of care and contributed to delay in definitive patient care. Furthermore, XRs ordered for evaluation of knee pain lacked the gold standard views in this study. There is a strong need to educate the providers, particularly VAMC nonorthopedic physicians, on when it is appropriate to order an MRI for evaluation of knee pain, in order to optimize the use of VAMC resources and deliver care in a time-efficient manner.

\section{Funding}

This work was supported in part by Career Development Award Number IK2 BX004879 from the United States Department of Veterans Affairs Biomedical Laboratory R\&D (BLRD) Service.

\section{Conflict of Interest}

F.H. 3rd has a grant with Arthrex, which is not relevant to this work. The remaining author do not report any conflicts of interests.

\section{References}

1 Maradit Kremers H, Larson DR, Crowson CS, et al. Prevalence of total hip and knee replacement in the United States. J Bone Joint Surg Am 2015;97(17):1386-1397

2 Dominick KL, Golightly YM, Jackson GL. Arthritis prevalence and symptoms among US non-veterans, veterans, and veterans 
receiving Department of Veterans Affairs Healthcare.J Rheumatol 2006;33(02):348-354

3 Losina E, Paltiel AD, Weinstein AM, et al. Lifetime medical costs of knee osteoarthritis management in the United States: impact of extending indications for total knee arthroplasty. Arthritis Care Res (Hoboken) 2015;67(02):203-215

4 Ma VY, Chan L, Carruthers KJ. Incidence, prevalence, costs, and impact on disability of common conditions requiring rehabilitation in the United States: stroke, spinal cord injury, traumatic brain injury, multiple sclerosis, osteoarthritis, rheumatoid arthritis, limb loss, and back pain. Arch Phys Med Rehabil 2014;95(05): 986-995.e1

5 Weinstein AM, Rome BN, Reichmann WM, et al. Estimating the burden of total knee replacement in the United States. J Bone Joint Surg Am 2013;95(05):385-392

6 Kellgren JH, Lawrence JS. Radiological assessment of osteo-arthrosis. Ann Rheum Dis 1957;16(04):494-502

7 Altman R, Asch E, Bloch D, et al; Diagnostic and Therapeutic Criteria Committee of the American Rheumatism Association. Development of criteria for the classification and reporting of osteoarthritis. Classification of osteoarthritis of the knee. Arthritis Rheum 1986;29(08):1039-1049

8 Vignon E, Conrozier T, Piperno M, Richard S, Carrillon Y, Fantino O. Radiographic assessment of hip and knee osteoarthritis. Recommendations: recommended guidelines. Osteoarthritis Cartilage 1999;7(04):434-436

9 Duncan ST, Khazzam MS, Burnham JM, Spindler KP, Dunn WR, Wright RW. Sensitivity of standing radiographs to detect knee arthritis: a systematic review of Level I studies. Arthroscopy 2015;31(02):321-328

10 Babatunde OM, Danoff JR, Patrick DA Jr, Lee JH, Kazam JK, Macaulay W. The Combination of the Tunnel View and WeightBearing Anteroposterior Radiographs Improves the Detection of Knee Arthritis. Arthritis (Egypt) 2016;2016:9786924

11 Jazrawi LM, Alaia MJ, Chang G, Fitzgerald EF, Recht MP. Advances in magnetic resonance imaging of articular cartilage. J Am Acad Orthop Surg 2011;19(07):420-429

12 Iglehart JK. The new era of medical imaging-progress and pitfalls. N Engl J Med 2006;354(26):2822-2828

13 Solivetti FM, Guerrisi A, Salducca N, et al. Appropriateness of knee MRI prescriptions: clinical, economic and technical issues. Radiol Med (Torino) 2016;121(04):315-322

14 Emery DJ, Shojania KG, Forster AJ, Mojaverian N, Feasby TE. Overuse of magnetic resonance imaging. JAMA Intern Med 2013;173(09):823-825

15 The American College of Radiology. 2010 ACR-SSR practice guideline for the performance and interpretation of magnetic resonance imaging (MRI) of the knee. Avaialble at: https://www. acr.org/-/media/ACR/Files/Practice-Parameters/mr-knee.pdf

16 Sherman SL, Gulbrandsen TR, Lewis HA, et al. Overuse of magnetic resonance imaging in the diagnosis and treatment of moderate to severe osteoarthritis. Iowa Orthop J 2018;38:33-37

17 Englund M, Guermazi A, Gale D, et al. Incidental meniscal findings on knee MRI in middle-aged and elderly persons. N Engl J Med 2008;359(11):1108-1115

18 Liu TC, Leung N, Edwards L, Ring D, Bernacki E, Tonn MD. Patients older than 40 years with unilateral occupational claims for new shoulder and knee symptoms have bilateral MRI changes. Clin Orthop Relat Res 2017;475(10):2360-2365

19 Song YD, Jain NP, Kim SJ, et al. Is knee magnetic resonance imaging overutilized in current practice? Knee Surg Relat Res 2015;27 (02):95-100
20 Fox MG, Chang EY, Amini B, et al; Expert Panel on Musculoskeletal Imaging. ACR appropriateness criteria chronic knee pain. J Am Coll Radiol 2018;15(11S):S302-S312

21 Perera NS, Joel J, Bunola JA. Anterior cruciate ligament rupture: delay to diagnosis. Injury 2013;44(12):1862-1865

22 Sathiyakumar V, Jahangir AA, Mir HR, et al. Patterns of costs and spending among orthopedic surgeons across the United States: a national survey. Am J Orthop 2014;43(01):E7-E13

23 Issa K, Jauregui JJ, McElroy M, Banerjee S, Kapadia BH, Mont MA. Unnecessary magnetic resonance imaging of hips: an economic burden to patients and the healthcare system. J Arthroplasty 2014;29(10):1911-1914

24 Wylie JD, Makarewich CA, Working ZM, et al. Findings associated with knee pathology on MRI in patients without osteoarthritis. J Am Acad Orthop Surg 2017;25(11):780-786

25 Amin N, Mclntyre L, Carter T, Xerogeanes J, Voigt J. Cost-effectiveness analysis of needle arthroscopy versus magnetic resonance imaging in the diagnosis and treatment of meniscal tears of the knee. Arthroscopy 2019;35(02):554-562.e13

26 Bradley SM, O'Donnell CI, Grunwald GK, et al. Facility-level variation in hospitalization, mortality, and costs in the 30 days after percutaneous coronary intervention: insights on short-term healthcare value from the Veterans Affairs Clinical Assessment, Reporting, and Tracking System (VA CART) Program. Circulation 2015;132(02):101-108

27 Taylor BC, Hagel Campbell E, Nugent S, et al. Three year trends in veterans health administration utilization and costs after traumatic brain injury screening among veterans with mild traumatic brain injury. J Neurotrauma 2017;34(17):2567-2574

28 Schweizer ML, Cullen JJ, Perencevich EN, Vaughan Sarrazin MS. Costs associated with surgical site infections in Veterans Affairs Hospitals. JAMA Surg 2014;149(06):575-581

29 Lee D, Begley CE. Delays in seeking health care: comparison of veterans and the general population. J Public Health Manag Pract 2017;23(02):160-168

30 Valsangkar NP, Eppstein AC, Lawson RA, Taylor AN. Effect of lean processes on surgical wait times and efficiency in a tertiary care Veterans Affairs Medical Center. JAMA Surg 2017;152(01): 42-47

31 Stroupe KT, Martinez R, Hogan TP, et al. Experiences with the Veterans' Choice Program. J Gen Intern Med 2019;34(10): 2141-2149

32 Bernstein J, Cain EL, Kneeland JB, Dalinka MK. The incidence of pathology detected by magnetic resonance imaging of the knee: differences based on the specialty of the requesting physician. Orthopedics 2003;26(05):483-485

33 van Oudenaarde K, Swart NM, Bloem JL, et al. General practitioners referring adults to MR imaging for knee pain: a randomized controlled trial to assess cost-effectiveness. Radiology 2018; 288(01):170-176

34 DAMASK (Direct Access to Magnetic Resonance Imaging: Assessment for Suspect Knees) Trial Team. Cost-effectiveness of magnetic resonance imaging of the knee for patients presenting in primary care. Br J Gen Pract 2008;58(556):e10-e16

35 Bryan S, Weatherburn G, Bungay H, et al. The cost-effectiveness of magnetic resonance imaging for investigation of the knee joint. Health Technol Assess 2001;5(27):1-95

36 Damen J, Schiphof D, Wolde ST, Cats HA, Bierma-Zeinstra SM, Oei EH. Inter-observer reliability for radiographic assessment of early osteoarthritis features: the CHECK (cohort hip and cohort knee) study. Osteoarthritis Cartilage 2014;22(07): 969-974 